IJMMS 2003:2, 87-96

PII. S0161171203201150

http://ijmms.hindawi.com

(c) Hindawi Publishing Corp.

\title{
BOUNDEDNESS FOR MULTILINEAR MARCINKIEWICZ OPERATORS ON CERTAIN HARDY SPACES
}

\author{
LIU LANZHE
}

Received 14 January 2002

The boundedness for the multilinear Marcinkiewicz operators on certain Hardy and Herz-Hardy spaces are obtained.

2000 Mathematics Subject Classification: 42B25, 42B20.

1. Introduction and definitions. Suppose that $S^{n-1}$ is the unit sphere of $\mathbb{R}^{n}$ $(n \geq 2)$ equipped with normalized Lebesgue measure $d \sigma=d \sigma\left(x^{\prime}\right)$. Let $\Omega$ be homogeneous of degree zero and satisfy the following two conditions:

(i) $\Omega(x)$ is continuous on $S^{n-1}$ and satisfies the Li $p_{\gamma}$ condition on $S^{n-1}$ $(0 \leq \gamma \leq 1)$, that is,

$$
\left|\Omega\left(x^{\prime}\right)-\Omega\left(y^{\prime}\right)\right| \leq M\left|x^{\prime}-y^{\prime}\right|^{\gamma}, \quad x^{\prime}, y^{\prime} \in S^{n-1}
$$

(ii) $\int_{S^{n-1}} S\left(x^{\prime}\right) d x^{\prime}=0$.

Let $m$ be a positive integer and $A$ be a function on $\mathbb{R}^{n}$. The multilinear Marcinkiewicz integral operator is defined by

$$
\mu_{\Omega}^{A}(f)(x)=\left[\int_{0}^{\infty}\left|F_{t}^{A}(f)(x)\right|^{2} \frac{d t}{t^{3}}\right]^{1 / 2},
$$

where

$$
\begin{gathered}
F_{t}^{A}(f)(x)=\int_{|x-y| \leq t} \frac{\Omega(x-y)}{|x-y|^{n-1}} \frac{R_{m+1}(A ; x, y)}{|x-y|^{m}} f(y) d y, \\
R_{m+1}(A ; x, y)=A(x)-\sum_{|\alpha| \leq m} \frac{1}{\alpha !} D^{\alpha} A(y)(x-y)^{\beta} .
\end{gathered}
$$

We denote that $F_{t}(f)(x)=f_{|x-y| \leq t}\left(\Omega(x-y) /|x-y|^{n-1}\right) f(y) d y$. We also denote that

$$
\mu_{\Omega}(f)(x)=\left(\int_{0}^{\infty}\left|F_{t}(f)(x)\right|^{2} \frac{d t}{t^{3}}\right)^{1 / 2},
$$

which is the Marcinkiewicz integral operator (see [5, 6, 12]). 
Note that when $m=0, \mu_{\Omega}^{A}$ is just the commutator of Marcinkiewicz operator (see [5, 12]). It is well known that multilinear operators are of great interest in harmonic analysis and have been widely studied by many authors (see [1, 2, $3,4,5]$ ). The main purpose of this paper is to consider the continuity of the multilinear Marcinkiewicz operators on certain Hardy and Herz-Hardy spaces. We first introduce some definitions (see $[7,8,9,10,11]$ ).

DEFINITION 1.1. Let $A$ be a function on $\mathbb{R}^{n}, m$ a positive integer, and $0<$ $p \leq 1$. A bounded measurable function $a$ on $\mathbb{R}^{n}$ is said to be a $\left(p, D^{m} A\right)$-atom if

(i) $\operatorname{supp} a \subset B=B\left(x_{0}, r\right)$,

(ii) $\|a\|_{L^{\infty}} \leq|B|^{-1 / p}$,

(iii) $\int a(y) d y=\int a(y) D^{\alpha} A(y) d y=0,|\alpha|=m$.

A temperate distribution $f$ is said to belong to $H_{D^{m_{A}}}^{p}\left(\mathbb{R}^{n}\right)$, if, in the Schwartz distributional sense, it can be written as

$$
f(x)=\sum_{j=0}^{\infty} \lambda_{j} a_{j}(x),
$$

where $a_{j}$ 's are $\left(p, D^{m} A\right)$-atoms, $\lambda_{j} \in C$, and $\sum_{j=0}^{\infty}\left|\lambda_{j}\right|^{p}<\infty$. Moreover, $\|f\|_{H_{D m_{A}}^{p}\left(\mathbb{R}^{n}\right)} \sim\left(\sum_{j=0}^{\infty}\left|\lambda_{j}\right|^{p}\right)^{1 / p}$.

Let $B_{k}=\left\{x \in \mathbb{R}^{n}:|x| \leq 2^{k}\right\}, C_{k}=B_{k} \backslash B_{k-1}, k \in \mathbb{Z}$, and $m_{k}(\lambda, f)=\mid\{x \in$ $\left.C_{k}:|f(x)|>\lambda\right\} \mid$; for $k \in \mathbb{N}$, let $\tilde{m}_{k}(\lambda, f)=m_{k}(\lambda, f)$ and $\tilde{m}_{0}(\lambda, f)=\mid\left\{x \in B_{0}\right.$ : $|f(x)|>\lambda\} \mid$.

DEFINITION 1.2. Let $0<p, q<\infty$, and $\alpha \in \mathbb{R}$.

(1) The homogeneous Herz space is defined by

$$
\dot{K}_{q}^{\alpha, p}=\left\{f \in L_{\mathrm{loc}}^{q}\left(\mathbb{R}^{n} \backslash\{0\}\right):\|f\|_{\dot{K}_{q}^{\alpha, p}\left(\mathbb{R}^{n}\right)}<\infty\right\},
$$

where

$$
\|f\|_{\dot{K}_{q}^{\alpha, p}\left(\mathbb{R}^{n}\right)}=\left[\sum_{k=-\infty}^{\infty} 2^{k \alpha p}\left\|f \chi_{k}\right\|_{L^{q}}^{p}\right]^{1 / p} .
$$

(2) The nonhomogeneous Herz space is defined by

$$
K_{q}^{\alpha, p}\left(\mathbb{R}^{n}\right)=\left\{f \in L_{\mathrm{loc}}^{q}\left(\mathbb{R}^{n}\right):\|f\|_{K_{q}^{\alpha, p}\left(\mathbb{R}^{n}\right)}<\infty\right\},
$$

where

$$
\|f\|_{K_{q}^{\alpha, p}\left(\mathbb{R}^{n}\right)}=\left[\sum_{k=1}^{\infty} 2^{k \alpha p}\left\|f \chi_{k}\right\|_{L^{q}}^{p}+\left\|f \chi_{B_{0}}\right\|_{L^{q}}^{p}\right]^{1 / p},
$$


where

$$
\|f\|_{W K_{q}^{\alpha, p}\left(\mathbb{R}^{n)}\right.}=\sup _{\lambda>0} \lambda\left[\sum_{k=0}^{\infty} 2^{k \alpha p} \tilde{m}_{k}(\lambda, f)^{p / q}\right]^{1 / p} .
$$

DEFINITION 1.3. Let $m$ be a positive integer and $A$ a function on $\mathbb{R}^{n}, \alpha \in \mathbb{R}$, and $1<q \leq \infty$. A function $a(x)$ on $\mathbb{R}^{n}$ is called a central $\left(\alpha, q, D^{m} A\right)$-atom (or a central $\left(\alpha, q, D^{m} A\right)$-atom of restrict type), if

(1) supp $a \subset B(0, r)$ for some $r>0$ (or for some $r \geq 1$ ),

(2) $\|a\|_{L^{q}} \leq|B(0, r)|^{-\alpha / n}$,

(3) $\int a(x) d x=\int a(x) D^{\beta} A(x) d x=0,|\beta|=m$.

A temperate distribution $f$ is said to belong to $H \dot{K}_{q, D^{m} A}^{\alpha, p}\left(\mathbb{R}^{n}\right)\left(\right.$ or $\left.H K_{q, D^{m}}^{\alpha, p}\left(\mathbb{R}^{n}\right)\right)$ if it can be written as $f=\sum_{j=-\infty}^{\infty} \lambda_{j} a_{j}$ (or $f=\sum_{j=0}^{\infty} \lambda_{j} a_{j}$ ) in the $S^{\prime}\left(\mathbb{R}^{n}\right)$ sense, where $a_{j}$ is a central $\left(\alpha, q, D^{m} A\right)$-atom (or a central $\left(\alpha, q, D^{m} A\right)$-atom of restrict type) supported on $B\left(0,2^{j}\right)$ and $\sum_{j=-\infty}^{\infty}\left|\lambda_{j}\right|^{p}<\infty$ (or $\left.\sum_{j=0}^{\infty}\left|\lambda_{j}\right|^{p}<\infty\right)$. Moreover, $\|f\|_{H \dot{K}_{q, D}^{\alpha, p} m_{A}}\left(\right.$ or $\left.\|f\|_{H K_{q, D}^{\alpha, p} m_{A}}\right) \sim\left(\sum_{j}\left|\lambda_{j}\right|^{p}\right)^{1 / p}$.

2. Theorems and proofs. We begin with some preliminary lemmas.

LEMmA 2.1 (see [2]). Let $A$ be a function on $\mathbb{R}^{n}$ and $D^{\alpha} A \in L^{q}\left(\mathbb{R}^{n}\right)$ for $|\alpha|=$ $m$ and some $q>n$. Then,

$$
\left|R_{m}(A: x, y)\right| \leq C|x-y|^{m} \sum_{|\alpha|=m}\left(\frac{1}{|\tilde{Q}(x, y)|} \int_{\tilde{Q}(x, y)}\left|D^{\alpha} A(z)\right|^{q} d z\right)^{1 / q}
$$

where $\tilde{Q}$ is the cube centered at $x$ and having side length $5 \sqrt{n}|x-y|$.

LEMMA 2.2. Let $1<p<\infty$ and $D^{\alpha} A \in L^{r}\left(\mathbb{R}^{n}\right),|\alpha|=m, 1<r \leq \infty$, and $1 / q=1 / p+1 / r$. Then, $\mu_{\Omega}^{A}$ is bound from $L^{p}\left(\mathbb{R}^{n}\right)$ to $L^{q}\left(\mathbb{R}^{n}\right)$, that is,

$$
\left\|\mu_{\Omega}^{A}(f)\right\|_{L^{q}} \leq C \sum_{|\alpha|=m}\left\|D^{\alpha} A\right\|_{L^{r}}\|f\|_{L^{p}}
$$

Proof. By Minkowski inequality and the condition of $\Omega$, we have

$$
\begin{aligned}
\mu_{\Omega}^{A}(f)(x) & \leq \int_{\mathbb{R}^{n}} \frac{|f(y)|\left|R_{m+1}(A ; x, y)\right|}{|x-y|^{m}}\left(\int_{|x-y|}^{\infty} \frac{d t}{t^{3}}\right)^{1 / 2} d y \\
& \leq C \int_{\mathbb{R}^{n}} \frac{\left|R_{m+1}(A ; x, y)\right|}{|x-y|^{m+n}}|f(y)| d y .
\end{aligned}
$$

Thus, the lemma follows from [3, 4]. 
THEOREM 2.3. Let $1 \geq p>n /(n+\gamma)$, and let $D^{\beta} A \in \operatorname{BMO}\left(\mathbb{R}^{n}\right)$ for $|\beta|=m$. Then, $\mu_{\Omega}^{A}$ is bounded from $H_{D^{m_{A}}}^{p}\left(\mathbb{R}^{n}\right)$ to $L^{p}\left(\mathbb{R}^{n}\right)$.

Proof. It suffices to show that there exists a constant $c>0$ such that, for every $\left(p, D^{m} A\right)$-atom $a$,

$$
\left\|\mu_{\Omega}^{A}(a)\right\|_{L^{p}} \leq C
$$

Let $a$ be a $\left(p, D^{m} A\right)$-atom supported on a ball $B=B\left(x_{0}, r\right)$. We write

$$
\begin{aligned}
\int_{\mathbb{R}^{n}}\left[\mu_{\Omega}^{A}(a)(x)\right]^{p} d x= & \int_{\left|x-x_{0}\right| \leq 2 r}\left[\mu_{\Omega}^{A}(a)(x)\right]^{p} d x \\
& +\int_{\left|x-x_{0}\right|>2 r}\left[\mu_{\Omega}^{A}(a)(x)\right]^{p} d x \\
& \equiv I+I I .
\end{aligned}
$$

For $I$, taking $q>1$ and by Hölder's inequality and the $L^{q}$-boundedness of $\mu_{\Omega}^{A}$ (see Lemma 2.2), we see that

$$
\begin{aligned}
I & \leq C\left\|\mu_{\Omega}^{A}(a)\right\|_{L^{q}}^{p} \cdot\left|B\left(x_{0}, 2 r\right)\right|^{1-p / q} \\
& \leq C\|a\|_{L^{q}|B|^{1-p / q}}^{p} \\
& \leq C .
\end{aligned}
$$

To obtain the estimate of $I I$, we need to estimate $\mu_{\Omega}^{A}(a)(x)$ for $x \in(2 B)^{c}$. Let $\tilde{B}=5 \sqrt{n} B$, and let $\tilde{A}(x)=A(x)-\sum_{|\alpha|=m}(1 / \alpha !)\left(D^{\alpha} A\right)_{\tilde{B}} \cdot x^{\alpha}$. Then, $R_{m}(A ; x, y)=$ $R_{m}(\tilde{A} ; x, y)$. By the vanishing moment of $a$, we write

$$
\begin{aligned}
F_{t}^{A}(a)(x)= & \int_{|x-y| \leq t}\left[\frac{\Omega(x-y)}{|x-y|^{m+n-1}}-\frac{\Omega\left(x-x_{0}\right)}{\left|x-x_{0}\right|^{m+n-1}}\right] R_{m}(\tilde{A} ; x, y) a(y) d y \\
& +\int_{|x-y| \leq t} \frac{\Omega\left(x-x_{0}\right)}{\left|x-x_{0}\right|^{m+n-1}}\left[R_{m}(\tilde{A} ; x, y)-R_{m}\left(\tilde{A} ; x, x_{0}\right)\right] a(y) d y \\
& -\sum_{|\alpha|=m} \frac{1}{\alpha !} \int_{|x-y| \leq t} \frac{\Omega(x-y)(x-y)^{\alpha}}{|x-y|^{m+n-1}}\left(D^{\alpha} A(y)-\left(D^{\alpha} A\right)_{B}\right) a(y) d y,
\end{aligned}
$$


thus,

$$
\begin{aligned}
\mu_{\Omega}^{A}(a)(x) \leq & {\left[\int _ { 0 } ^ { \infty } \left(\int_{|x-y| \leq t}\left|\frac{\Omega(x-y)}{|x-y|^{m+n-1}}-\frac{\Omega\left(x-x_{0}\right)}{\left|x-x_{0}\right|^{m+n-1}}\right|\right.\right.} \\
& \left.\left.\times\left|R_{m}(\tilde{A} ; x, y)\right||a(y)| d y\right)^{2} \frac{d t}{t^{3}}\right]^{1 / 2} \\
& +\left[\int _ { 0 } ^ { \infty } \left(\int_{|x-y| \leq t} \frac{\left|\Omega\left(x-x_{0}\right)\right|}{\left|x-x_{0}\right|^{m+n-1}}\right.\right. \\
& +\left[\int_{0}^{\infty} \mid \sum_{|\alpha|=m} \frac{1}{\alpha !} \int_{|x-y| \leq t} \frac{\Omega(x-y)(x-y)^{\alpha}}{|x-y|^{m+n-1}}\right. \\
& \left.\left.\left.\quad I I_{1}+I I_{2}+I I_{3} . \quad \times\left.\left(D^{\alpha} A(y)-\left(D^{\alpha} A\right)_{B}\right) a(y) d y\right|^{2} \frac{d t}{t^{3}}\right]^{1 / 2}\left(\tilde{A} ; x, x_{0}\right)|| a(y) \mid d y\right)^{2} \frac{d t}{t^{3}}\right]^{1 / 2}
\end{aligned}
$$

By Lemma 2.1, for $y \in B$ and $x \in 2^{k+1} B \backslash 2^{k} B$, we know

$$
\left|R_{m}(\tilde{A} ; x, y)\right| \leq C|x-y|^{m} \sum_{|\alpha|=m}\left|D^{\alpha} A(x)-\left(D^{\alpha} A\right)_{2^{k} B}\right|
$$

By the condition of $\Omega$ and Minkowski's inequality, and noting that $|x-y| \sim$ $\left|x-x_{0}\right|$ for $y \in B$ and $x \in \mathbb{R}^{n} \backslash B$, we obtain

$$
\left|\frac{\Omega(x-y)}{|x-y|^{m+n-1}}-\frac{\Omega\left(x-x_{0}\right)}{\left|x-x_{0}\right|^{m+n-1}}\right| \leq C\left(\frac{r}{\left|x-x_{0}\right|^{m+n}}+\frac{r^{\gamma}}{\left|x-x_{0}\right|^{m+n+\gamma-1}}\right) .
$$

Thus,

$$
\begin{aligned}
I_{1} \leq & C \int_{B}\left|R_{m}(\tilde{A} ; x, y)\right||a(y)|\left(\int_{|x-y|}^{\infty} \frac{d t}{t^{3}}\right)^{1 / 2} \\
& \times\left(\frac{r}{\left|x-x_{0}\right|^{m+n}}+\frac{r^{\gamma}}{\left|x-x_{0}\right|^{m+n+\gamma-1}}\right) d y \\
\leq & C\left(\frac{r}{\left|x-x_{0}\right|^{n+1}}+\frac{r^{\gamma}}{\left|x-x_{0}\right|^{n+\gamma}}\right)|B|^{1-1 / p} \sum_{|\alpha|=m}\left|D^{\alpha} A(x)-\left(D^{\alpha} A\right)_{2^{k} B}\right| .
\end{aligned}
$$


On the other hand, by the following formula (see [2]):

$$
R_{m}(\tilde{A} ; x, y)-R_{m}\left(\tilde{A} ; x, x_{0}\right)=\sum_{|\beta|<m} \frac{1}{\beta !} R_{m-|\beta|}\left(D^{\beta} \tilde{A} ; y, x_{0}\right)\left(x-x_{0}\right)^{\beta}
$$

and Lemma 2.1, we get

$$
\begin{aligned}
\mid R_{m}(\tilde{A} ; x, y) & -R_{m}\left(\tilde{A} ; x, x_{0}\right) \mid \\
& \leq C \sum_{|\beta|<m} \sum_{|\alpha|=m}\left|x_{0}-y\right|^{m-|\beta|}\left|x-x_{0}\right|^{|\beta|}|| D^{\alpha} A \|_{\text {BMO }}
\end{aligned}
$$

so that

$$
\begin{aligned}
I I_{2} \leq & C \int_{B}\left|x-x_{0}\right|^{-(n+m)} \sum_{|\beta|<m}\left|R_{m-|\beta|}\left(D^{\beta} \tilde{A} ; y, x_{0}\right)\right|\left|x-x_{0}\right|^{|\beta|}|a(y)| d y \\
\leq & C \int_{B}\left|x-x_{0}\right|^{-(n+m)} \sum_{|\beta|<m}\left|x_{0}-y\right|^{m-|\beta|}\left|x-x_{0}\right|^{|\beta|} \\
& \times \sum_{|\alpha|=m}\left\|D^{\alpha} A\right\|_{\text {BMO }}|a(y)| d y \\
\leq & C \sum_{|\alpha|=m}\left\|D^{\alpha} A\right\|_{\text {BMO }} \int_{B} \frac{\left|x_{0}-y\right|}{\left|x-x_{0}\right|^{n+1}}|a(y)| d y \\
\leq & C \sum_{|\alpha|=m}\left\|D^{\alpha} A\right\|_{\text {BMO }}\left|x-x_{0}\right|^{-n-1}|B|^{1 / n-1 / p+1} .
\end{aligned}
$$

For $I_{3}$, and by the vanishing moment of $a$, we write,

$$
\begin{aligned}
& \int \frac{\Omega(x-y)(x-y)^{\alpha}}{|x-y|^{m}}\left(D^{\alpha} A(y)-\left(D^{\alpha} A\right)_{B}\right) a(y) d y \\
& =\int\left[\frac{\Omega(x-y)(x-y)^{\alpha}}{|x-y|^{m}}-\frac{\Omega\left(x-x_{0}\right)\left(x-x_{0}\right)^{\alpha}}{\left|x-x_{0}\right|^{m+n-1}}\right]\left[D^{\alpha} A(y)-\left(D^{\alpha} A\right)_{B}\right] a(y) d y .
\end{aligned}
$$

Similar to the estimate of $I I_{1}$, we obtain

$$
\begin{aligned}
I_{3} \leq & C \sum_{|\alpha|=m}\left(\frac{r}{\left|x-x_{0}\right|^{n+1}}+\frac{r^{\gamma}}{\left|x-x_{0}\right|^{n+\gamma}}\right) \\
& \times \int_{B}\left|x_{0}-y\right|\left|D^{\alpha} A(y)-\left(D^{\alpha} A\right)_{B}\right||a(y)| d y \\
\leq & \left.C \sum_{|\alpha|=m}|| D^{\alpha} A\left|\|_{\mathrm{BMO}}\right| B\right|^{1-1 / p}\left(\frac{r}{\left|x-x_{0}\right|^{n+1}}+\frac{r^{\gamma}}{\left|x-x_{0}\right|^{n+\gamma}}\right) .
\end{aligned}
$$


Recalling that $p>n /(n+\gamma)$, therefore,

$$
\begin{aligned}
I I \leq & \sum_{k=1}^{\infty} \int_{2^{k+1} B \backslash 2^{k} B}\left[\mu_{\Omega}^{A}(a)(x)\right]^{p} d x \\
\leq & C \sum_{k=1}^{\infty} \int_{2^{k+1} B \backslash 2^{k} B}\left(\frac{r}{\left|x-x_{0}\right|^{n+1}}+\frac{r^{\gamma}}{\left|x-x_{0}\right|^{n+\gamma}}\right)^{p}|B|^{p-1} \\
& \times\left(\sum_{|\alpha|=m}\left|D^{\alpha} A(x)-\left(D^{\alpha} A\right)_{2^{k+1} B}\right|\right)^{p} d x \\
& +C\left(\sum_{|\alpha|=m}\left\|D^{\alpha} A\right\|_{\mathrm{BMO}}\right)^{p} \sum_{k=1}^{\infty} \int_{2^{k+1} B \backslash 2^{k} B}\left|x-x_{0}\right|^{-p(n+1)}|B|^{p(1+1 / n-1 / p)} d x \\
\leq & C\left(\sum_{|\alpha|=m}\left\|D^{\alpha} A\right\|_{\mathrm{BMO}}\right)^{p} \sum_{k=1}^{\infty}\left(2^{k(n-p-p n)}+2^{k(n-p n-p \gamma)}\right) \\
\leq & C\left(\sum_{|\alpha|=m}\left\|D^{\alpha} A\right\|_{\mathrm{BMO}}\right)^{p},
\end{aligned}
$$

which, together with the estimate for $I$, yields the desired result. This finishes the proof of Theorem 2.3.

THEOREM 2.4. Let $0<p<\infty, 1<q<\infty, n(1-1 / q) \leq \alpha<n(1-1 / q)+\gamma$, and $D^{\beta} A \in \operatorname{BMO}\left(\mathbb{R}^{n}\right)$ for $|\beta|=m$. Then, $\mu_{\Omega}^{A}$ is bounded from $H \dot{K}_{q, D^{m} A}^{\alpha, p}\left(\mathbb{R}^{n}\right)$ to $\dot{K}_{q}^{\alpha, p}\left(\mathbb{R}^{n}\right)$.

Proof. Let $f \in H \dot{K}_{q, D^{m} A}^{\alpha, p}\left(\mathbb{R}^{n}\right)$ and $f(x)=\sum_{j=-\infty}^{\infty} \lambda_{j} a_{j}(x)$ be the atomic decomposition for $f$ as in Definition 1.3. We write

$$
\begin{aligned}
\left\|\mu_{\Omega}^{A}(f)\right\|_{\dot{K}_{q}^{\alpha, p}\left(\mathbb{R}^{n}\right)} \leq & C\left[\sum_{k=-\infty}^{\infty} 2^{k \alpha p}\left(\sum_{j=-\infty}^{k-3}\left|\lambda_{j}\right|\left\|\mu_{\Omega}^{A}\left(a_{j}\right) \chi_{k}\right\|_{L^{q}}\right)^{p}\right]^{1 / p} \\
& +C\left[\sum_{k=-\infty}^{\infty} 2^{k \alpha p}\left(\sum_{j=k-2}^{\infty}\left|\lambda_{j}\right|\left\|\mu_{\Omega}^{A}\left(a_{j}\right) \chi_{k}\right\|_{L^{q}}\right)^{p}\right]^{1 / p} \\
\equiv & I+I I .
\end{aligned}
$$

For $I I$, and by the boundedness of $\mu_{\Omega}^{A}$ on $L^{q}\left(\mathbb{R}^{n}\right)$ (see Lemma 2.2), we have

$$
I I \leq C\left[\sum_{k=-\infty}^{\infty} 2^{k \alpha p}\left(\sum_{j=k-2}^{\infty}\left|\lambda_{j}\right|\left\|a_{j}\right\|_{L^{q}}\right)^{p}\right]^{1 / p}
$$




$$
\begin{aligned}
& \leq C\left[\sum_{k=-\infty}^{\infty} 2^{k \alpha p}\left(\sum_{j=k-2}^{\infty}\left|\lambda_{j}\right| 2^{-j \alpha}\right)^{p}\right]^{1 / p} \\
& \leq C \begin{cases}{\left[\sum_{k=-\infty}^{\infty} 2^{k \alpha p} \sum_{j=k-2}^{\infty}\left|\lambda_{j}\right|^{p} 2^{-j \alpha p}\right]^{1 / p},} & 0<p \leq 1 \\
{\left[\sum_{k=-\infty}^{\infty} 2^{k \alpha p}\left(\sum_{j=k-2}^{\infty}\left|\lambda_{j}\right|^{p} 2^{-j \alpha p / 2}\right)\left(\sum_{j=k-2}^{\infty} 2^{-j \alpha p^{\prime} / 2}\right)^{p / p^{\prime}}\right]^{1 / p},} & p>1\end{cases} \\
& \leq C \begin{cases}{\left[\sum_{j=-\infty}^{\infty}\left|\lambda_{j}\right|^{p}\left(\sum_{k=-\infty}^{j+2} 2^{(k-j) \alpha p}\right)\left|\lambda_{j}\right|^{p} 2^{-j \alpha p}\right]^{1 / p},} & 0<p \leq 1 \\
{\left[\sum_{j=-\infty}^{\infty}\left|\lambda_{j}\right|^{p}\left(\sum_{k=-\infty}^{j+2} 2^{(k-j) \alpha p / 2}\right)\right]^{1 / p},} & p>1\end{cases} \\
& \leq C\left(\sum_{j=-\infty}^{\infty}\left|\lambda_{j}\right|^{p}\right)^{1 / p} \leq C\|f\|_{H \dot{K}_{q, D}^{\alpha, p} m_{A}\left(\mathbb{R}^{n}\right)} .
\end{aligned}
$$

For $I$, and similar to the proof of Theorem 2.3, we have, for $x \in C_{k}, j \leq k-3$,

$$
\begin{aligned}
\mu_{\Omega}^{A}\left(a_{j}\right)(x) \leq & C\left(|x|^{-n-m-1}\left|B_{j}\right|^{1 / n}+|x|^{-n-m-\gamma}\left|B_{j}\right|^{\gamma / n}\right) \\
& \times \\
& \left(\int_{B_{j}}\left|a_{j}(y)\right|\left|R_{m}(\tilde{A} ; x, y)\right| d y\right) \\
& +\left.C \sum_{|\beta|=m}|| D^{\beta} A\right|_{\mathrm{BMO}}|x|^{-n-1}\left|B_{j}\right|^{1 / n} \int_{B_{j}}|a(y)| d y \\
+ & C\left(|x|^{-n-1}\left|B_{j}\right|^{1+1 / n}+|x|^{-n-\gamma}\left|B_{j}\right|^{1+\gamma / n}\right) \\
& \times \sum_{|\beta|=m} \int_{B_{j}}\left|D^{\beta} A(y)-\left(D^{\beta} A\right)_{B_{j}}\right||a(y)| d y \\
\leq & C\left(2^{-k(n+1)} 2^{j(1+n(1-1 / q)-\alpha)}+2^{-k(n+\gamma)} 2^{j(\gamma+n(1-1 / q)-\alpha)}\right) \\
& \times\left(\sum_{|\beta|=m}\left|D^{\beta} A(x)-\left(D^{\beta} A\right)_{B_{k}}\right|\right) \\
& +C \sum_{|\alpha|=m}\left\|D^{\alpha} A\right\|_{\mathrm{BMO}}(k-j) \\
& \times\left(2^{-k(n+1)} 2^{j(1+n(1-1 / q)-\alpha)}+2^{-k(n+\gamma)} 2^{j(\gamma+n(1-1 / q)-\alpha)}\right)
\end{aligned}
$$


thus,

$$
\begin{aligned}
& I \leq C\left[\sum _ { k = - \infty } ^ { \infty } 2 ^ { k \alpha p } \left(\sum_{j=-\infty}^{k-3}\left|\lambda_{j}\right|\left(2^{-k(n+1)+j(1+n(1-1 / q)-\alpha)}+2^{-k(n+\gamma)+j(\gamma+n(1-1 / q)-\alpha)}\right)\right.\right. \\
& \left.\left.\left.\quad \times \sum_{|\alpha|=m}\left(\int_{B_{k}}\left|D^{\alpha} A(x)-\left(D^{\alpha} A\right)_{B_{k}}\right| q\right)\right)^{1 / q}\right)^{p}\right]^{1 / p} \\
& +C\left[\sum _ { k = - \infty } ^ { \infty } 2 ^ { k \alpha p } \left(\sum _ { j = - \infty } ^ { k - 3 } | \lambda _ { j } | ( k - j ) \left(2^{-k(n+1)+j(1+n(1-1 / q)-\alpha)}\right.\right.\right. \\
& \left.\left.\left.+2^{-k(n+\gamma)+j(\gamma+n(1-1 / q)-\alpha)}\right) 2^{k n / q} \sum_{|\alpha|=m}\left\|D^{\alpha} A\right\|_{\mathrm{BMO}}\right)^{p}\right]^{1 / p} \\
& \equiv I_{1}+I_{2} .
\end{aligned}
$$

To estimate $I_{1}$ and $I_{2}$, we consider two cases.

CASE $1(0<p \leq 1)$. We have

$$
\begin{aligned}
I_{1} & \leq C\left[\sum _ { k = - \infty } ^ { \infty } 2 ^ { k \alpha p } \sum _ { j = - \infty } ^ { k - 3 } | \lambda _ { j } | ^ { p } \left(2^{[-k(n+1)+j(1+n(1-1 / q)-\alpha)] p}\right.\right. \\
& \left.\left.+2^{[-k(n+\gamma)+j(\gamma+n(1-1 / q)-\alpha)] p}\right) 2^{k n p / q}\left(\sum_{|\beta|=m}\left\|D^{\beta} A\right\|_{\mathrm{BMO}}\right)^{p}\right]^{1 / p} \\
& =C \sum_{|\beta|=m}\left\|D^{\beta} A\right\|_{\mathrm{BMO}}\left[\sum_{j=-\infty}^{\infty}\left|\lambda_{j}\right|^{p} \sum_{k=j+3}^{\infty}\left(2^{(j-k)(1+n(1-1 / q)-\alpha) p}+2^{(j-k)(\gamma+n(1-1 / q)-\alpha) p}\right)\right]^{1 / p} \\
& \leq C \sum_{|\beta|=m}\left\|D^{\beta} A\right\|_{\mathrm{BMO}}\left(\sum_{j=-\infty}^{\infty}\left|\lambda_{j}\right|^{p}\right)^{1 / p} \\
& \leq C\|f\|_{H \dot{K}_{q, D}^{\alpha, p} m_{A}\left(\mathbb{R}^{n}\right)} .
\end{aligned}
$$

Similarly,

$$
I_{2} \leq C\|f\|_{H \dot{K}_{q, D}^{\alpha, p} m_{A}\left(\mathbb{R}^{n}\right)}
$$


CASE $2(p>1)$. By Hölder's inequality, we deduce that

$$
\begin{aligned}
I_{1} \leq C \sum_{|\beta|=m}\left\|D^{\beta} A\right\|_{\mathrm{BMO}}\left[\sum_{j=-\infty}^{\infty}\left(\sum_{j=-\infty}^{k-3}\left|\lambda_{j}\right|^{p} 2^{(j-k) p(\gamma+n(1-1 / q)-\alpha) / 2}\right)\right. \\
\left.\quad \times\left(\sum_{j=-\infty}^{k-3} 2^{(j-k) p^{\prime}(\gamma+n(1-1 / q)-\alpha) / 2}\right)^{p / p^{\prime}}\right]^{1 / p} \\
\leq C\left(\sum_{j=-\infty}^{\infty}\left|\lambda_{j}\right|^{p}\right)^{1 / p} \leq C\|f\|_{H \dot{K}_{q, D}^{\alpha, p} m_{A}\left(\mathbb{R}^{n}\right)}, \\
I_{2} \leq C\|f\|_{H \dot{K}_{q, D^{m} m_{A}\left(\mathbb{R}^{n}\right)}^{\alpha, p}}
\end{aligned}
$$

This finishes the proof of Theorem 2.

REMARK 2.5. Theorem 2.4 also holds for nonhomogeneous Herz-type space.

\section{REFERENCES}

[1] J. Cohen and J. A. Gosselin, On multilinear singular integrals on $\mathbf{R}^{n}$, Studia Math. 72 (1982), no. 3, 199-223.

[2] _ A BMO estimate for multilinear singular integrals, Illinois J. Math. 30 (1986), no. 3, 445-464.

[3] Y. Ding, A note on multilinear fractional integrals with rough kernel, Adv. Math. (China) 30 (2001), no. 3, 238-246.

[4] Y. Ding and S. Lu, Weighted boundedness for a class of rough multilinear operators, Acta Math. Sin. (Engl. Ser.) 17 (2001), no. 3, 517-526.

[5] Y. Ding, S. Lu, and Q. Xue, On Marcinkiewicz integral with homogeneous kernels, J. Math. Anal. Appl. 245 (2000), no. 2, 471-488.

[6] Y. Ding and Q. Xue, Commutator of Marcinkiewicz integral on Hardy space, preprint.

[7] G. Hu, S. Lu, and D. C. Yang, The applications of weak Herz spaces, Adv. in Math. (China) 26 (1997), no. 5, 417-428.

[8] _ The weak Herz spaces, Beijing Shifan Daxue Xuebao 33 (1997), no. 1, 27-34.

[9] S. Lu and D. C. Yang, The decomposition of weighted Herz space on $\mathbf{R}^{n}$ and its applications, Sci. China Ser. A 38 (1995), no. 2, 147-158.

[10] _ The weighted Herz-type Hardy space and its applications, Sci. China Ser. A 38 (1995), no. 6, 662-673.

[11] _ The continuity of commutators on Herz-type spaces, Michigan Math. J. 44 (1997), no. 2, 255-281.

[12] A. Torchinsky and S. L. Wang, A note on the Marcinkiewicz integral, Colloq. Math. 60/61 (1990), no. 1, 235-243.

Liu Lanzhe: Department of Applied Mathematics, Hunan University, Changsha 410082, China

E-mail address: 1anzhe1iu@263.net 


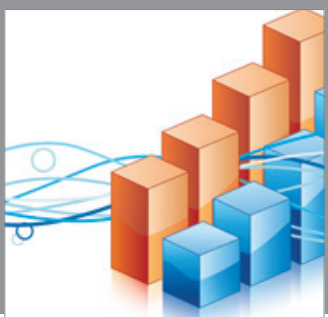

Advances in

Operations Research

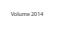

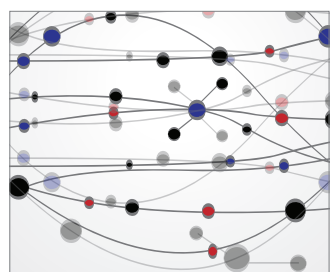

\section{The Scientific} World Journal
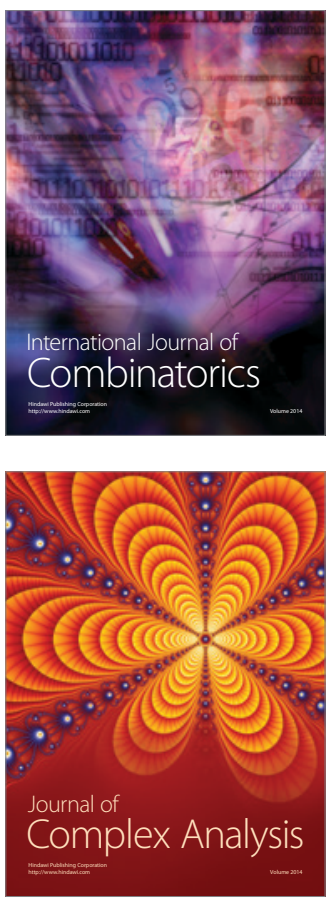

International Journal of

Mathematics and

Mathematical

Sciences
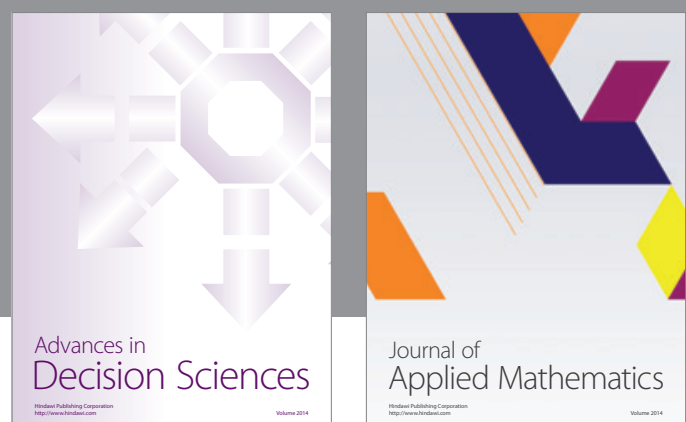

Journal of

Applied Mathematics
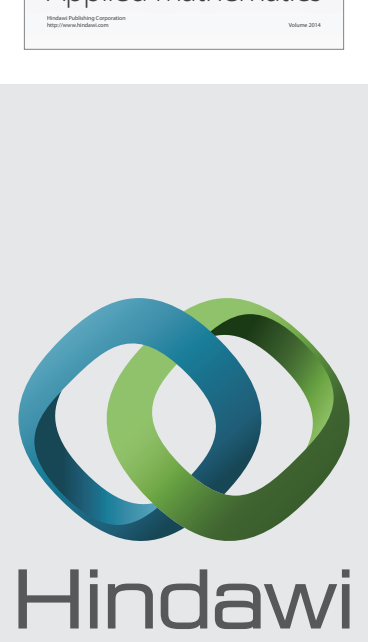

Submit your manuscripts at http://www.hindawi.com
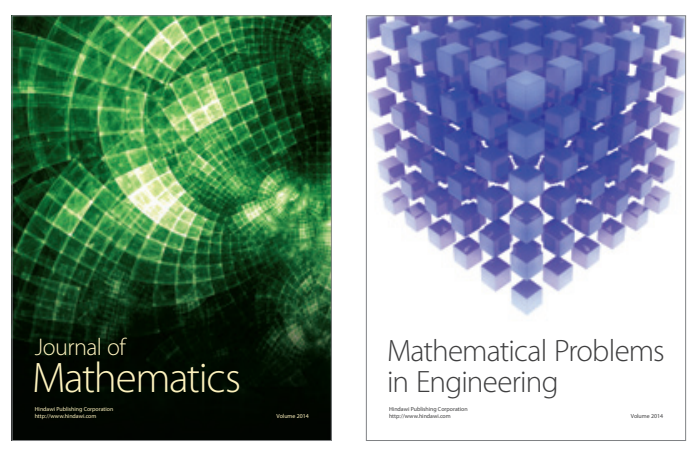

Mathematical Problems in Engineering
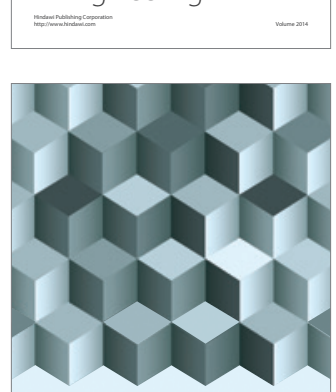

Journal of

Function Spaces
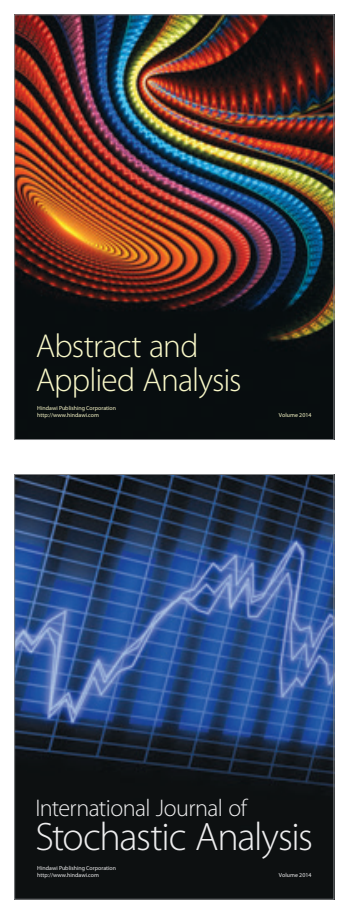

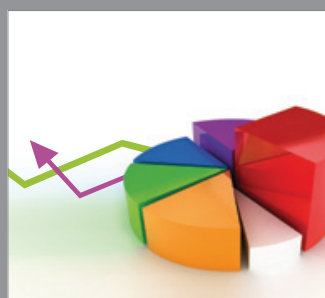

ournal of

Probability and Statistics

Promensencen
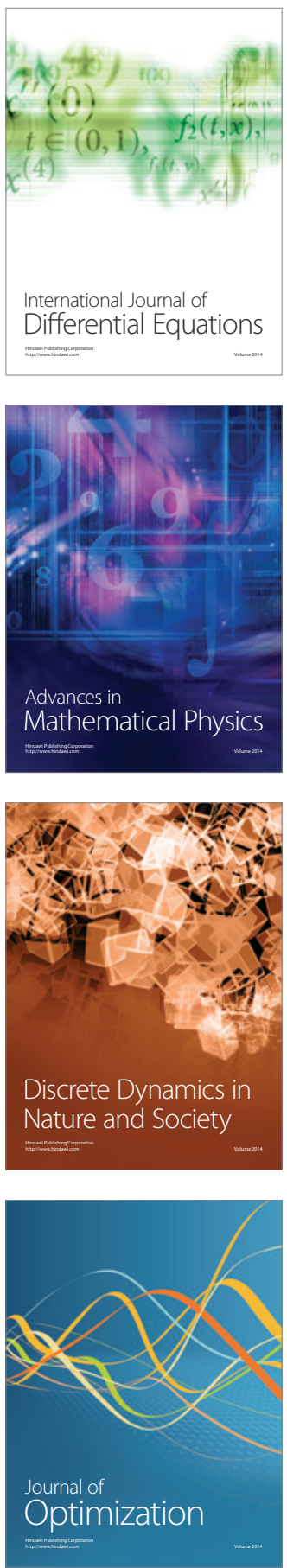